\title{
BROADENING OF SPECTRAL LINES DUE TO DYNAMIC MULTIPLE SCATTERING AND THE TULLY-FISHER RELATION
}

\author{
Sisir Roy ${ }^{1,2}$, \& Menas Kafatos ${ }^{1}$ \\ Center for Earth Observing and Space Research \\ Institute for Computational Sciences and Informatics and \\ Department of Physics, George Mason University \\ Fairfax, VA 22030 USA \\ Suman Dutta ${ }^{2}$ \\ Physics and Applied Mathematics Unit \\ Indian Statistical Institute, Calcutta, INDIA \\ 1,2 e.mail: sroy2@osf1.gmu.edu \\ ${ }^{1}$ e.mail : mkafatos@compton.gmu.edu \\ 2 email : res9428@isical.ac.in
}

\begin{abstract}
The frequency shift of spectral lines is most often explained by the Doppler Effect in terms of relative motion, whereas theDoppler broadening of a particular line mainly depends on the absolute temperature. The Wolf effect on the other hand deals with the correlation induced spectral change and explains both the broadening and shift of the spectral lines. In this framework a relation between the width of the spectral line is related to the redshift $z$ for the line and hence with the distance. For smaller values of $z$ a relation similar to the Tully-Fisher relation can be obtained and for larger values of $z$ a more general relation can be constructed. The derivation of this kind of relation based on dynamic multiple scattering theory may play a significant role in explaining the overall spectra of quasi stellar objects. We emphasize that this mechanism is not applicable for nearby galaxies, $z \leq 1$.
\end{abstract}

Keywords : spectral line broadening, spectral line shift, Tully-Fisher relation.

PACS : 32.70.Jz 


\section{Introduction}

In studying the motion of astronomical objects, astrophysicists utilize the study of frequency shift of spectral lines. In general, an emphasis on relating the shift of a spectral line with its width has not been made so far. The Tully-Fisher relation ${ }^{(1)}$ is an empirical correlation which finds that the luminosity $L$ of a disk galaxy is proportional to its maxium rotational velocity $V_{\max }^{\alpha}$, where $\alpha$ has been observationally established to be $(3-4)^{(2)}$. In spite of the frequenty use of the TF relation as a distance indicator, the physical origin of this relationship is poorly understood, and it remains unclear whether all rotationally supported disk galaxies, including late-type spirals and irregulars, obey a single luminosity-line width correlation. Recently, Matthews et al (3) made an attempt to analyze this situation. It is generally argued that in order to obtain a measure of the maximum rotational velocity of a disk from its measured global $H-I$ profile width, some correction to the observed line width should be made for the effects of broadening due to turbulent (i.e. non-rotational) motions ( cf. Roberts ${ }^{(4)}$, Bottinelli et al. (5) $)$. A linear summation of rotational and random motions adequately describes the observed profile widths of giant galaxies, while for dwarfs galaxies( i.e. slowly rotating galaxies with Gaussian-like line profiles ), a sum in the quadrature of the random and rotational terms is appropriate. However, Rhee ${ }^{(6)}$ has pointed out the shortcomings of this type of addition for the type of objects considered here.

On the other hand, a dynamic multiple scattering theory ${ }^{(7),(8)}$ derived from the field of Statistical Optics has been developed to account for the shift of a spectral line as well as the broadening of the line. It is shown that when light passes through a turbulent(or inhomogeneous ) medium, due to multiple scattering the shift and the width can be calculated. Here, a sufficient condition for redshift has been derived and when applicable the shift is shown to be larger than broadening. The width of the spectral line can be calculated after multiple scatterings and a relation can be derived between the width and the shift $z$ which applies to any value of $z$. For small values of $z$ this can be reduced to a Tully-Fisher type of relation. It would be interesting to estimate this width and make a comparison with the correction part of the line profile width considerd by Bottinelli et $\mathrm{al}^{(5)}$ applying to non-rotational motion. We want to 
emphasize that it is then possible to derive a relation for distance indicator ( which reduces to a Tully-Fisher type relation as a special case ) by studying the particular collisional mechanism which itself is a physical process. This kind of physical process can be identified even in the laboratory experiments and as such it is plausible that it applies to astronomical objects.

In this paper we shall start with a brief discussion of the various type of broadenings (section 2). In section 3 we shall discuss the main results of our Multiple Scattering Theory within the Wolf framework of redshifts ${ }^{(9)}$. Based on this approach a relation between the shfit $z$ and the width will be derived in section 4 . Finally, the possible implications for QSO observations will be discussed in section 5 .

\section{Spectral Broadening}

If a spectral line is examined by means of a spectrograph, its width is dependent on the slit width employed. In general, the narrower the slit width the less broad is the resulting spectral line as recorded on a photographic plate. Nevertheless, however narrow the slit width is, the sharpest line has a finite width even for the best optical system. Three causes producing the breadth of a spectral line are :

1. Its natural width

2. Doppler effect

3. external effect

\subsection{Natural Line Width}

An atom can stay in two type of states. A state in which an atom free from external effects can remain for an arbitrarily long time is called a stationary state. Only the ground state is stationary since it corresponds to the minimum possible energy for a given atom. An excited state is non-stationary since spontaneous transitions to lower energy levels are possible for it. Such states are called transient states which are characterized by a finite life time. A quantitative measure of instability of an excited 
state is the time $\tau$ during which the number of atoms in a system at a given excited state decreases by a factor of e. This quantity $\tau$ is known as the lifetime of the excited state and coincides with the average time spent by the atoms in the excited state. In quantum mechanics, $\tau$ is associated with the probability of spontaneous radiative transitions from a given excited state to a lower energy level. Life times of excited states of atoms generally lie in the range $10^{-9}$ to $10^{-8}$ second. The finiteness of the life time of an atom in a transient state can easily be taken into account by introducing the damping factor into the expression for the wave function

$$
\psi(\vec{r}, t)=\exp ^{\left[-\frac{i}{h} E t-\frac{\gamma t}{2}\right]}
$$

where $\gamma$ is a positive constant. It should be noted that the wave function in stationary state is

$$
\psi(\vec{r}, t)=\exp ^{\left[-\frac{i}{h} E t\right]} \psi(\vec{r})
$$

Therefore the above function not only oscillates with a frequency $\omega=\frac{E}{h}$, but also attenuates with time due to the presence of the factor $\exp ^{\left[-\frac{\gamma t}{2}\right]}$. The probability density of finding the atom in the state $\psi$ is given by

$$
|\psi(\vec{r}, t)|^{2}=\exp [-\gamma t]|\psi(\vec{r})|^{2}
$$

The time during which the probability density diminishes to $\frac{1}{e}$ th of its initial value is obviously the life time $\tau$ of the state. It then follows that

$$
\gamma \tau=1
$$

The quantity $\gamma$ defined by this expression is called the damping constant.

Since energy and time are canonically conjugate quantities, according to the Heisenberg Uncertainty relation, the energy of an excited state is not exactly definite. The indeterminacy $\Gamma$ in the energy of a transient state is connected with its lifetime $\tau$ through the relation

$$
\Gamma \sim \frac{\hbar}{\tau}=\hbar \gamma
$$

Blurring of adjacent energy levels can generally be due to various reasons. The quantity $\Gamma$ associated with the probability of spontaneous radiative transitions is called 
the natural width of the level. If an atom remains in the normal state for a long time the uncertainty of the energy value is small and the level is sharp. If the electron is excited to an upper level where it remains for some time, the uncertainty of the energy value is greater and the width of the level is greater. The natural line width is the minimum limit for the radiation line width.

\subsection{Doppler Broadening}

The thermal motion of emitting atoms leads to the so-called Doppler broadening of spectral lines. Owing to thermal agitation, most of the atoms emitting light have high velocities. The random motion of the atoms and the molecules in a gas, however, produce a net broadening of the lines with no apparent shift in its central maximum. The frequency spread of this line is called the half-intensity breadth, and is given by

$$
\delta=1.67 \frac{\nu_{0}}{c} \sqrt{\frac{2 R T}{m}}
$$

The half intensity breadth is defined as the interval between two points where the intensity drops to half its maximum vale. Here, $R$ is the gas constant, $T$ is the temperature of the gas in $\mathrm{K}$, and $m$ is the atomic weight. Therefore the Doppler broadening is

1. proportional to the frequency $\nu_{0}$

2. proportional to the square root of the (absolute) temperature $T$; and

3. inversely proportional to the square root of the atomic weight $m$.

Experimental observations indicate that in keeping with the above equation, in order to produce sharper lines in any given spectrum, the temperature must be lowered. Furthermore, for a given temperature the lines produced by the lighter elements in the periodic table are in general broader than those produced by the heavy elements.

A comparison of the estimated values of natural width and Doppler width shows that the Doppler width at room temperatures is much larger than the natural width [ Figure $1]$. 


\subsection{Collisional Broadening}

The spectral broadenings described in the previous two subsections, can be classified as due to internal effects. There exist other reasons for spectral line broadening specifically an excited atom in a gas with a finite number density of particles undergoes collisions with neighbouring atoms. Since the phase of radiation changes upon each collision, the monochromatic nature of the emission line is violated. This is effectively taken into account by introducing the total level width, which is equal to $\Gamma+\Gamma_{\text {col }}$, where

$\Gamma_{\text {col }}=\frac{1}{\pi \tau_{0}}$ is the collision level width ( $\tau_{0}$ is the mean free time of the atom in gaseous medium). Another mechanism, which can explain the line broadening, is an application of statistical optics where the existence of a random dielectric susceptibility which fluctuates both spatially and temporally is assumed. The scattering of light induced by the random susceptibilty can produce both a shift and a broadening of the spectral line. This is called the Wolf effect ${ }^{(9)}$, the main features of which are described as follows.

\section{Dynamic Multiple Scattering Theory}

First, we briefly state the main results of Wolf's scattering mechanism. Let us consider a polychromatic electromagnetic field of light of central frequency $\omega_{0}$ and width $\delta_{0}$, incident on the scatterer. The incident spectrum is assumed to be of the form

$$
S^{(i)}(\omega)=A_{0} e^{\left[-\frac{1}{2 \delta_{0}^{2}}\left(\omega-\omega_{0}\right)^{2}\right]}
$$

The spectrum of the scattered field is given by ${ }^{(10)}$

$$
S^{(\infty)}\left(r \overrightarrow{u^{\prime}}, \omega^{\prime}\right)=A \omega^{\prime 4} \int_{-\infty}^{\infty} K\left(\omega, \omega^{\prime}, \vec{u}, \overrightarrow{u^{\prime}}\right) S^{(i)}(\omega) d \omega
$$

which is valid within the first order Born approximation ${ }^{(11)}$. Here $K\left(\omega, \omega^{\prime}\right)$ is the so called scattering kernel and it plays the most important role in this mechanism. $\vec{u}$ and $\overrightarrow{u^{\prime}}$ are the unit vectors in the direction of incident and scattered fields respectively. Instead of studying $\mathcal{K}\left(\omega, \omega^{\prime}\right)$ in detail, we consider a particular case for the correlation function $G(\vec{R}, T ; \omega)$ of the generalized dielectric susceptibility $\eta(\vec{r}, t ; \omega)$ of the medium 
which is characterized by an anisotropic Gaussian function

$$
\begin{aligned}
G(\vec{R}, T ; \omega) & =<\eta^{*}(\vec{r}+\vec{R}, t+T ; \omega) \eta(\vec{r}, t ; \omega)> \\
& =G_{0} \exp \left[-\frac{1}{2}\left(\frac{X^{2}}{\sigma_{x}^{2}}+\frac{Y^{2}}{\sigma_{y}^{2}}+\frac{Z^{2}}{\sigma_{z}^{2}}+\frac{c^{2} T^{2}}{\sigma_{\tau}^{2}}\right)\right]
\end{aligned}
$$

Here $G_{0}$ is a positive constant, $\vec{R}=(X, Y, Z)$, and $\sigma_{x}, \sigma_{y}, \sigma_{z}, \sigma_{\tau}$ are correlation lengths. The anisotropy is indicated by the unequal correlation lengths in different spatial as well as temporal directions. $\mathcal{K}\left(\omega, \omega^{\prime}\right)$ can be obtained from the four dimensional Fourier Transform of the correlation function $G(\vec{R}, T ; \omega)$. In this case $\mathcal{K}\left(\omega, \omega^{\prime}\right)$ can be shown to be of the form

$$
\mathcal{K}\left(\omega, \omega^{\prime}\right)=\operatorname{Bexp}\left[-\frac{1}{2}\left(\alpha^{\prime} \omega^{\prime 2}-2 \beta \omega \omega^{\prime}+\alpha \omega^{2}\right)\right]
$$

where

$$
\begin{aligned}
& \alpha \quad=\frac{\sigma_{x}^{2}}{c^{2}} u_{x}^{2}+\frac{\sigma_{y}^{2}}{c^{2}} u_{y}^{2}+\frac{\sigma_{z}^{2}}{c^{2}} u_{z}^{2}+\frac{\sigma_{\tau}^{2}}{c^{2}} \\
& \alpha^{\prime} \quad=\frac{\sigma_{x}^{2}}{c^{2}} u_{x}^{\prime 2}+\frac{\sigma_{y}^{2}}{c^{2}} u_{y}^{\prime 2}+\frac{\sigma_{z}^{2}}{c^{2}} u_{z}^{\prime 2}+\frac{\sigma_{\tau}^{2}}{c^{2}} \\
& \text { and } \beta=\frac{\sigma_{x}^{2}}{c^{2}} u_{x} u_{x}^{\prime}+\frac{\sigma_{y}^{2}}{c^{2}} u_{y} u_{y}^{\prime}+\frac{\sigma_{z}^{2}}{c^{2}} u_{z} u_{z}^{\prime}+\frac{\sigma_{\tau}^{2}}{c^{2}}
\end{aligned}
$$

Here $\hat{u}=\left(u_{x}, u_{y}, u_{z}\right)$ and $\hat{u}^{\prime}=\left(u_{x}^{\prime}, u_{y}^{\prime}, u_{z}^{\prime}\right)$ are the unit vectors in the directions of the incident and scattered fields respectively.

Substituting (1) \& (4) in (2), we finally get

$$
S^{(\infty)}\left(\omega^{\prime}\right)=A^{\prime} e^{\left[-\frac{1}{2 \delta_{0}^{\prime 2}}\left(\omega^{\prime}-\bar{\omega}_{0}\right)^{2}\right]}
$$

where

$$
\left.\begin{array}{ll}
\bar{\omega}_{0} & =\frac{|\beta| \omega_{0}}{\alpha^{\prime}+\delta_{0}^{2}\left(\alpha \alpha^{\prime}-\beta^{2}\right)} \\
\delta_{0}^{\prime 2} & =\frac{\alpha \delta_{0}^{2}+1}{\alpha^{\prime}+\delta_{0}^{2}\left(\alpha \alpha^{\prime}-\beta^{2}\right)} \\
\text { and } A^{\prime} & =\sqrt{\frac{\pi}{2\left(\alpha \delta_{0}^{2}+1\right)}} A B A_{0} \omega_{0}^{\prime 4} \delta_{0} \exp \left[\frac{|\beta| \omega_{0} \bar{\omega}_{0}-\alpha \omega_{0}^{2}}{2\left(\alpha \delta_{0}^{2}+1\right)}\right]
\end{array}\right\}
$$

Though $A^{\prime}$ depends on $\omega^{\prime}$, it was approximated by James and Wolf ${ }^{(8)}$ to be a constant so that $S^{(\infty)}\left(\omega^{\prime}\right)$ can be considered to be Gaussian. 
The relative frequency shift is defined as

$$
z=\frac{\omega_{0}-\bar{\omega}_{0}}{\bar{\omega}_{0}}
$$

where $\omega_{0}$ and $\bar{\omega}_{0}$ denote the unshifted and shifted central frequencies respectively. We say that the spectrum is redshifted or blueshifted according to whether $z>0$ or $z<0$ respectively. Here

$$
z=\frac{\alpha^{\prime}+\delta_{0}^{2}\left(\alpha \alpha^{\prime}-\beta^{2}\right)}{|\beta|}-1
$$

It is important to note that this $z$-number does not depend on the incident frequency, $\omega_{0}$. This is a very important aspect if the mechanism is to apply in the astronomical domain. Expression (15) implies that the spectrum can be shifted to the blue or to the red, according to the sign of the term $\alpha^{\prime}+\delta_{0}^{2}\left(\alpha \alpha^{\prime}-\beta^{2}\right)>|\beta|$. To obtain the no-blueshift condition, we use Schwarz's Inequality which implies that $\alpha \alpha^{\prime}-\beta^{2} \geq 0$. Thus, we can take

$$
\alpha^{\prime}>|\beta|
$$

as the sufficient condition to have only redshift by that mechanism.

Let's now assume that the light in its journey encounters many such scatterers. What we observe at the end is the light scattered many times, with an effect as that stated above in every individual process. Let there be N scatterers between the source and the observer and $z_{n}$ denote the relative frequency shift after the $n^{\text {th }}$ scattering of the incident light from the $(n-1)^{t h}$ scatterer, with $\omega_{n}$ and $\omega_{n-1}$ being the central frequencies of the incident spectra at $n^{t h}$ and $(n-1)^{t h}$ scatterers. Then by definition,

$$
z_{n}=\frac{\omega_{n-1}-\omega_{n}}{\omega_{n}}, \quad n=1,2, \ldots, N
$$

or,

$$
\frac{\omega_{n-1}}{\omega_{n}}=1+z_{n}, \quad n=1,2, \ldots, N
$$

Taking the product over $n$ from $n=1$ to $n=N$, we get,

$$
\frac{\omega_{0}}{\omega_{N}}=\left(1+z_{1}\right)\left(1+z_{2}\right) \ldots\left(1+z_{N}\right)
$$

The left hand side of the above equation is nothing but the ratio of the source frequency and the final or observed frequency $z_{f}$. Hence,

$$
1+z_{f}=\left(1+z_{1}\right)\left(1+z_{2}\right) \ldots\left(1+z_{N}\right)
$$


Since the $z$-number due to such effect does not depend upon the central frequency of the incident spectrum, each $z_{i}$ depends on $\delta_{i-1}$ only, not $\omega_{i-1}$ [here $\omega_{j}$ and $\delta_{j}$ denote the central frequency and the width of the incident spectrum at $(j+1)^{\text {th }}$ scatterer]. To find the exact dependence we first calculate the broadening of the spectrum after $\mathrm{N}$ number of scatterings.

\subsection{Effect of Multiple Scatterings on the Spectral Line Width}

From the second equation in (13), we can easily write,

$$
\left.\begin{array}{rl}
\delta_{n+1}^{2} & =\frac{\alpha \delta_{n}^{2}+1}{\alpha^{\prime}+\left(\alpha \alpha^{\prime}-\beta^{2}\right) \delta_{n}^{2}} \\
& =\left(\frac{\alpha \delta_{n}^{2}+1}{\alpha^{\prime}}\right)\left[1+\delta_{n}^{2}\left(\frac{\alpha \alpha^{\prime}-\beta^{2}}{\alpha^{\prime}}\right)\right]^{-1}
\end{array}\right\}
$$

From (13), we can also write

$$
\omega_{n+1}=\frac{\omega_{n}|\beta|}{\alpha^{\prime}+\left(\alpha \alpha^{\prime}-\beta^{2}\right) \delta_{n}^{2}}
$$

Then from (14) \& (15), we can write

$$
\left.\begin{array}{rl}
z_{n+1} & =\frac{\omega_{n}-\omega_{n+1}}{\omega_{n+1}} \\
& =\frac{\alpha^{\prime}+\left(\alpha \alpha^{\prime}-\beta^{2}\right) \delta_{n}^{2}}{|\beta|}-1 \\
& =\frac{\alpha^{\prime}}{|\beta|}\left\{1+\left(\frac{\alpha \alpha^{\prime}-\beta^{2}}{\alpha^{\prime}}\right) \delta_{n}^{2}\right\}-1
\end{array}\right\}
$$

Let's assume that the redshift per scattering process is very small, i.e.,

$$
0<\epsilon=z_{n+1} \quad<<1
$$

for all $n$.

Then,

$$
\begin{aligned}
1+\epsilon & =\frac{\alpha^{\prime}}{|\beta|}\left\{1+\left(\frac{\alpha \alpha^{\prime}-\beta^{2}}{\alpha^{\prime}}\right) \delta_{n}^{2}\right\} \\
\text { or, }(1+\epsilon) \frac{|\beta|}{\alpha^{\prime}} & =1+\left(\frac{\alpha \alpha^{\prime}-\beta^{2}}{\alpha^{\prime}}\right) \delta_{n}^{2}
\end{aligned}
$$


In order to satisfy this condition and in order to have a redshift only ( or positive z), we see that the first factor $\frac{\alpha^{\prime}}{|\beta|}$ in the right term cannot much bigger than 1, and, more important,

$$
\left(\frac{\alpha \alpha^{\prime}-\beta^{2}}{\alpha^{\prime}}\right) \delta_{n}^{2} \quad<\quad 1
$$

In that case, from (17), after neglecting higher order terms, the expression for $\delta_{n+1}^{2} \quad$ can be well approximated as:

$$
\delta_{n+1}^{2} \approx\left(\frac{\alpha \delta_{n}^{2}+1}{\alpha^{\prime}}\right)\left[1-\delta_{n}^{2}\left(\frac{\alpha \alpha^{\prime}-\beta^{2}}{\alpha^{\prime}}\right)\right]
$$

which, after carrying out a simplification, gives a very important recurrence relation:

$$
\delta_{n+1}^{2}=\frac{1}{\alpha^{\prime}}+\frac{\beta^{2}}{\alpha^{\prime 2}} \delta_{n}^{2}
$$

Therefore,

$$
\begin{aligned}
\delta_{n+1}^{2} & =\frac{1}{\alpha^{\prime}}+\frac{\beta^{2}}{\alpha^{\prime 2}} \delta_{n}^{2} \\
& =\frac{1}{\alpha^{\prime}}+\frac{\beta^{2}}{\alpha^{\prime 2}}\left[\frac{1}{\alpha^{\prime}}+\frac{\beta^{2}}{\alpha^{\prime 2}} \delta_{n-1}^{2}\right] \\
& =\left(\frac{\beta^{2}}{\alpha^{\prime 2}}\right)^{2} \delta_{n-1}^{2}+\frac{1}{\alpha^{\prime}}\left(1+\frac{\beta^{2}}{\alpha^{\prime 2}}\right) \\
& \left.\ldots \ldots \ldots . . . \ldots \frac{\beta^{2 n}}{\alpha^{\prime 2 n}}\right) . \\
& =\left(\frac{\beta^{2}}{\alpha^{\prime 2}}\right)^{n+1} \delta_{0}^{2}+\frac{1}{\alpha^{\prime}}\left(1+\frac{\beta^{2}}{\alpha^{\prime 2}}+\ldots\right.
\end{aligned}
$$

Thus

$$
\delta_{N+1}^{2}=\left(\frac{\beta^{2}}{\alpha^{\prime 2}}\right)^{N+1} \delta_{0}^{2}+\frac{1}{\alpha^{\prime}}\left(1+\frac{\beta^{2}}{\alpha^{\prime 2}}+\ldots \frac{\beta^{2 N}}{\alpha^{\prime 2 N}}\right) .
$$

As the number of scattering increases, the width of the spectrum obviously increases and the most important topic to be considered is whether this width is below some tolerance limit or not, from the observational point of view. There may be several measures of that tolerance limit. One of them is the Sharpness Ratio, defined as

$$
Q=\frac{\omega_{f}}{\delta_{f}}
$$

where $\omega_{f} \& \delta_{f}$ are the mean frequency \& the width of the observed spectrum. 
After $N$ number of scattering, this sharpness ratio, say $Q_{N}$, is given by the following recurrence relation :

$$
Q_{N+1}=Q_{N} \sqrt{\frac{\alpha^{\prime}}{\alpha^{\prime}+\left(\alpha \alpha^{\prime}-\beta^{2}\right) \delta_{N}^{2}}-\frac{1}{\alpha \delta_{N}^{2}+1}} .
$$

It is easy to verify that the expression under the square root lies between $0 \& 1$. Therefore, $Q_{N+1}<Q_{N}$, and the line is broadened as the scattering process goes on ( Figs.2 and 3).

Under the sufficient condition of redshift [i.e., $\left.|\beta|<\alpha^{\prime}\right]^{(12)}$ it was shown that in the observed spectrum

$$
\Delta \omega_{n+1} \gg \delta_{n}
$$

if the following condition holds:

$$
\frac{\delta_{n} \omega_{0}\left(\alpha \alpha^{\prime}-\beta^{2}\right)}{\alpha^{\prime}+\left(\alpha \alpha^{\prime}-\beta^{2}\right) \delta_{n}^{2}} \gg 1
$$

where $\omega_{0}$ is the source frequency.

The relation (23) signifies that the shift is more prominent than the effective broadening so that the spectral lines are observable and can be analyzed. If, on the other hand ,the broadening is higher than the shift of the spectral line, it will be impossible to detect the shift from the blurred spectrum. Hence we can take relation (24) to be one of the conditions necessary for the observed spectrum to be analyzable. For large $N$ (i.e., $N \rightarrow \infty$ ), the series in the second term of right hand side of (22) converges to a finite sum and we get

$$
\delta_{N+1}^{2}=\left(\frac{\beta^{2}}{\alpha^{\prime 2}}\right)^{N+1} \delta_{0}^{2}+\frac{\alpha^{\prime}}{\alpha^{\prime 2}-\beta^{2}} .
$$

If $\delta_{0}$ is considered as arising out of Doppler broadenning only, we can estimate $\delta_{\text {Dop }} \sim 10^{9}$ for $T=10^{4} \mathrm{~K}$. On the other hand, for anisotropic medium, we can take $\sigma_{x}=\sigma_{y}=3.42 \times 10^{-1}, \sigma_{z}=8.73 \times 10^{-1}, \alpha^{\prime}=8.68 \times 10^{-30}, \alpha=8.536 \times 10^{-30}$ and $\beta=$ $8.607 \times 10^{-30}$ for $\theta=15^{0(9)}$. Then the second term of the above expression will be much larger than the first term, and effectively, Doppler broadenning can be neglected in comparison to that due to multiple scattering effect.

Now if we consider the other condition, i.e., $\quad \alpha^{\prime}<|\beta|$, the series in (22) will be a divergent one and $\delta_{N+1}^{2}$ will be finitely large for large but finite $N$. However, if the 
condition (23) is to be satisfied, then the shift in frequency will be larger than the width of the spectral lines. In that case the condition $\alpha^{\prime}<|\beta|$ indicates that blueshift may also be observed but the width of the spectral lines can be large enough depending on how large the number of collisions is. So in general, the blueshifted lines should be of larger widths than redshifted lines and may not be as easily observable.

\subsection{Effect of Source Frequency on Broadening}

Rearranging equation (24) we get,

$$
\left(\delta_{n}-\frac{\omega_{0}}{2}\right)^{2} \ll \frac{\omega_{0}^{2}}{4}-\frac{\alpha^{\prime}}{\alpha \alpha^{\prime}-\beta^{2}}
$$

Since the left side is non-negative, the right side must be positive. Moreover, since the mean frequency of any source is always positive, i.e., $\omega_{0} \geq 0$, we must have

$$
\omega_{0} \gg \sqrt{\frac{4 \alpha^{\prime}}{\alpha \alpha^{\prime}-\beta^{2}}} .
$$

We take the right side of this inequality to be the critical source frequency $\omega_{\mathbf{c}}$ which is defined here as.

$$
\omega_{c}=\sqrt{\frac{4 \alpha^{\prime}}{\alpha \alpha^{\prime}-\beta^{2}}} .
$$

Thus for a particular medium between the source and the observer, the critical source frequency is the lower limit of the frequency of any source whose spectrum can be clearly analyzed. In other words, the shift of any spectral line from a source with frequency less than the critical source frequency for that particular medium cannot be detected due to its high broadening.

We now can classify the spectra of the different sources, from which light comes to us after passing through a scattering medium characterized by the parameters $\alpha, \alpha^{\prime}, \beta$. If we allow only small angle scattering in order to get prominent spectra, according to Wolf mechanism, they will either be blueshifted or redshifted. The redshift of spectral lines may or may not be detected according to whether the condition (24) does or does not hold. In this way those sources whose spectra are redshifted, are classified in two cases, viz., $\omega_{\mathbf{0}}>\omega_{\mathbf{c}}$ and $\omega_{0} \leq \omega_{c}$. In the first case, the shifts of the spectral 
lines can be easily detected due to condition (23). But in the later case, the spectra will suffer from the resultant blurring.

\subsection{Doppler Shift vs. Wolf Shift}

As we have seen in section(3.1) and section(3.2), the mean frequency and width of a spectral line change after each scattering. The changes are given by the recurrence relations:

$$
\delta_{n+1}^{2}=\frac{1}{\alpha^{\prime}}+\frac{\beta^{2}}{\alpha^{2}} \delta_{n}^{2}
$$

and

$$
\omega_{n+1}=\frac{\omega_{n}|\beta|}{\alpha^{\prime}+\left(\alpha \alpha^{\prime}-\beta^{2}\right) \delta_{n}^{2}} .
$$

Using these, we can easily find the Wolf-contribution to the observed shift assuming only that the source under consideration is quasi-monochromatic. For this we rewrite the equation $(21)$ as :

$$
\delta_{n}^{2}=\frac{\alpha^{2}}{\beta^{2}} \delta_{n+1}^{2}-\frac{\alpha^{\prime}}{\beta^{2}} .
$$

Now, according to Schrödinger ${ }^{(13)}$, the width of the spectral lines must increase as a result of collisional processes. If this is the sole reason for the broadening, then we can estimate $\mathrm{N}$, the number of collisions it undergoes in its way to us by comparing the width at each scatterer with a preassumed small $\delta$ [ the spectral width of the quasi-monochromatic source ]. We can easily calculate

$$
z_{W}=\left(1+z_{\text {scat }}\right)^{N}-1
$$

where $z_{\text {scat }}$ is the $z$-number due to small angle scattering at each scatterer. The following graph (Fig.4)illustrates the Wolf contribution in various observed shifts.

The solid line curve represents the Wolf-contribution, while the dotted line $(y=x)$ represents the observed $z$-number. Thus the difference in height of these two curves provides the contribution due to the Doppler effect which up to the present day was assumed to be the total contribution to $z$. 


\section{Width of the spectral lines and the Tully- Fisher Relation}

According to our result(13), we conclude that both the shift and the width of a spectral line depend on the medium parameters. In Fig.5, we show the variation of the spectral line width as a function of shift. We can write the relation between the width and the shift as

$$
W=K\left(a^{2}+\delta_{0}^{2} z^{2}\right)^{\frac{1}{2(1+z)}}
$$

where $K$ is a constant, $a^{2}$ is the minimum broadening and $\delta_{0}^{2}$ is the spectral width inherent to the source. The above relation is valid for $z>-1$. Taking the logarithm of both sides we can write

$$
\ln W=\frac{1}{2(1+z)} \ln K\left(a^{2}+\delta_{0}^{2} z^{2}\right)
$$

It is well known ${ }^{(14)}$ that the distance modulus $d$ can be written in terms of $z$ as

$$
d=m-M=42.38-5 \log \left(\frac{H}{100}\right)+5 \log z+(1.086)\left(1-q_{0}\right) z+O\left(z^{2}\right)
$$

Where $H$ is the Hubble constant in $\mathrm{km} / \mathrm{sec} / \mathrm{Mpc}$ and $M$ refer to the absolute magnitude. Now for small z i.e. $z<<1$,

$$
d=42.38-5 \log \left(\frac{H}{100}\right)+\frac{1.086}{2} z \quad \text { taking } \quad q_{0}=\frac{1}{2}
$$

After simplification we can write

$$
z=\frac{d-C}{.543} ; \quad \text { where } \quad C=42.38-5 \log \left(\frac{H}{100}\right)
$$

Now substituting this value of $z$ in equation(30) we can write

$$
d=-\frac{1.086}{\log E} \log W+N
$$

where

$$
\log E=\log \left(K a^{2}\right) \text { and } N=C+0.543
$$


The above relation between the distance modulus and the width has a striking similarity to the Tully-Fisher relation but without any angular dependence. The reason is obvious since we have considered the shift and width due to scatterings only without considering any rotational effects. It appears that in the case of photons which are emitted perpendicular to the plane of the galaxy, we will be observing those photons only without any rotational effects. It should be mentioned that Bottenelli et al. ${ }^{(4)}$ considered linear turbulence correction for the profile width as

$$
W_{20, i, c}=\frac{\left[W_{20, \mathrm{obsv}}-W_{t, 20}\right]}{\sin i}
$$

where $W_{20, \text { obsv }}$ is the observed (profile) width at 20 percent peak maximum, corrected for instrumental broadening, $W_{(t, 20)}$ is the correction term for turbulence and the factor $\sin i$ corrects for disk inclination.

It would be interesting to compare this contribution for galaxy spectra due to nonrotational part with the width calculated from miltiple scattering theory. These will be considered in a subsequent publication.

\section{Possible Implications}

Unlike the Doppler effect where the width of a line is unrelated to the shift, the Wolf mechanism predicts a tight relationship between the width and the shift of a line. As such, it is evident from the above analysis that Dynamic Multiple Scattering Theory within Wolf's framework might play a significant role for QSOs in the following ways.

1. The width and shifts of various spectral lines in different regions, say for UV, optical etc.,for AGNs and Quasars may be explained in a consistent manner if the information regarding the environments around these objects were available.

2. Since the Wolf contribution becomes prominent for larger $z$ as can be seen from Fig.2, this mechanism may shed new light in explaining the redshifts of quasars which tend to be large and peaking at $z \sim 2-3$ as well as for hight redshift Galaxies $(z>1)$. 
3. For blue shift (i.e., $\alpha^{\prime}<|\beta|$ ), as the broadenning is much larger, as is evident from (22), than the shift, one would get a continuous spectrum with no discrete lines.

4. For low redshifts we can derive a relation like the Tully-Fisher as a function for distance modulus and width given by a physical mechanism. We also derived a general relation for higher redshifts(eqn. (30)) which can be verified from observations. Further studies can be made regarding the theoretical basis of the Baldwin Effect ${ }^{(15)}$ for line and continuum correlations in AGN.

Lastly, we should mention that the critical source frequency relating to the screening effect which plays a crucial role in explaining both redhsift and spectral width can be tested in Laboratory Experiments.

Acknowledgements: One of the authors ( S.D.) greatly acknowledges World Laboratory, Laussane for financial support during this work and Prof. B. K. Datta ( Director, World Laboratory, Calcutta Branch ) for encouragement. The author(S.R.) is indebted to Prof. Jack Sulentic,University of Alabama, for valuable suggestions and comments. 


\section{REFERENCES}

1. Tully, R.B. and Fisher, J.R.(1977), A \& A, 54, 661.

2. Aaronson, M.,Huchra, J.P.,\& Mould, J.R.(1979), ApJ,229,1.

3. Matthews, L.D., Driel,van Driel \& Gallaagher,III J.S.(1998), "An Exploration of the Tully-Fisher for Extreme Late-Type Spiral Galaxies", astro-ph/9810042.

4. Roberts, M.S., AJ, (1978),83,1026.

5. Bottenelli, L.,Gouguenheim, L., Paturel,G.,de Vaucouleurs,G. (1983),A \& A , $118,4$.

6. Rhee,M.H. (1996),Ph.D. Thesis, University of Groningen.

7. Datta S, Roy S, Roy M \& Moles M, (1998), Int. Jour. of Theo. Phys., 37,N4, 1313.

8. Datta S, Roy S, Roy M \& Moles M, (1998), Int.Jour.Theort.Phys., 37,N5, 1469.

9. Wolf E. and James D.F.V.(1996),Rep. Progr. Phys.59, 771.

10. James D.F.V and Wolf E, (1990), Phys. Lett. A, 146, 167.

11. Born, M.\& Wolf,E. (1998), "Principle of Optics", 6th edition, Pergamon, Oxford.

12. Datta S, Roy S, Roy M and Moles M, (1998), Phys.Rev.A,58,720.

13. Schrödinger E., (1955), IL Nuovo Cimento, 1, 63.

14. Narlikar, J. V., (1983), "Introduction to Cosmology", Jones \& Bertlett Publishers, Inc., Boston.

15. Osmer, S.Patrick, Shields C.Joshep ,(1998), "A Review of Line and Continuum Relations in AGNs", astro-ph/9811459. 


\section{Figure Captions :}

Figure 1 : Doppler Broadening of Spectral Line.

Figure 2 :Variation of Sharpness of a Spectral Line with Relative Frequency Shift (for low $z$ ).

Figure 3 : Variation of Sharpness for high $(z)$.

Figure 4.Wolf Contribution in Relative Frequency Shift

Figure 5. Variation of Width with shift. 
Summary of the changes and response to all recommendations and criticisms

1. On page 3 : we have given the refernce of E.Wolf and D.V.F.James: Rep.Prog.Phys. 59, 771 (1996).

2. On page 6 : we have quoted the above reference as suggested by the referee.

3. In eqn(8) : we have rewritten the equation as per suggestion of the referee

$$
S^{(\infty)}\left(r \overrightarrow{u^{\prime}}, \omega^{\prime}\right)=A \omega^{4} \int_{-\infty}^{\infty} K\left(\omega, \omega^{\prime}, \vec{u}, \overrightarrow{u^{\prime}}\right) S^{(i)}(\omega) d \omega
$$

4. On page 8 : the refernce was given as James and Wolf instead of Wolf.

\section{Response to Valerie Miller regarding figure problems with MSAA7127}

All the necessary changes have been made as suggested by Valerie Miller. 
To

The Editor

Physical Review A

\section{Re : MS AA7127}

Dear Sir,

We are resubmitting our MS AA7127 ( Broadening of spectral lines.. by Roy, Kafatos and Dutta) considering all the recommendations of the referee for your consideration. We are enclosing a summary of the changes made in the text as well as the changes in the figures as suggested by Valerie Miller seperately. Moreover, we have typed the whole MS in double spaced style as suggested by the editor. Hope it will suffice your purpose.

With best regards,

Yours Sincerely, ( Sisir Roy).

Address for Communication :

Prof.Sisir Roy

Center for Earth Observing and Space Research

Institute of Computational Sciences and Informatics

George Mason University

Room 113, Science and Technology 1

Fairfax, VA 22030-4444

USA .

Fax : (703) 9933628. 


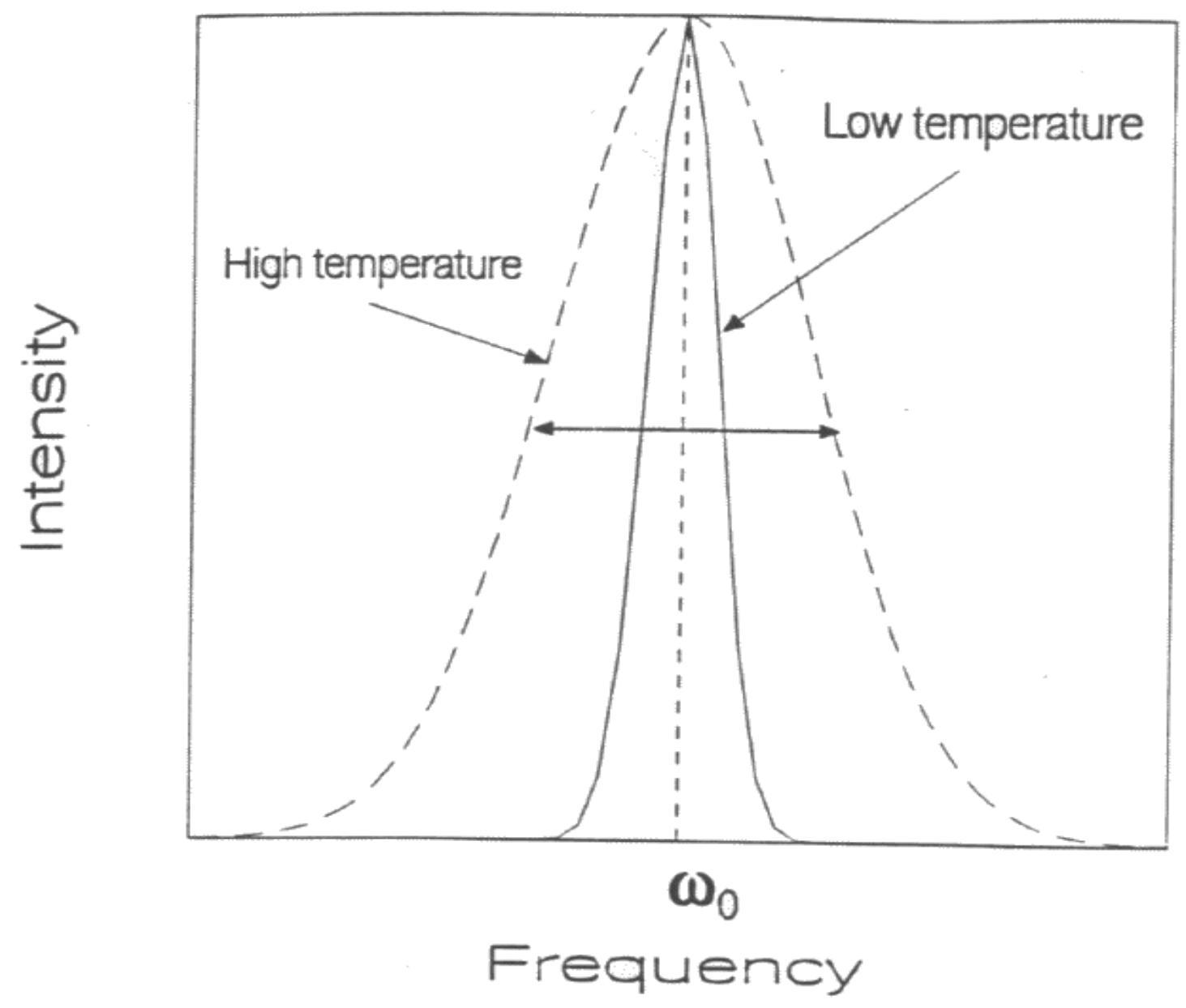

Fig. 1: Doppler Broadening of Spectral Line 


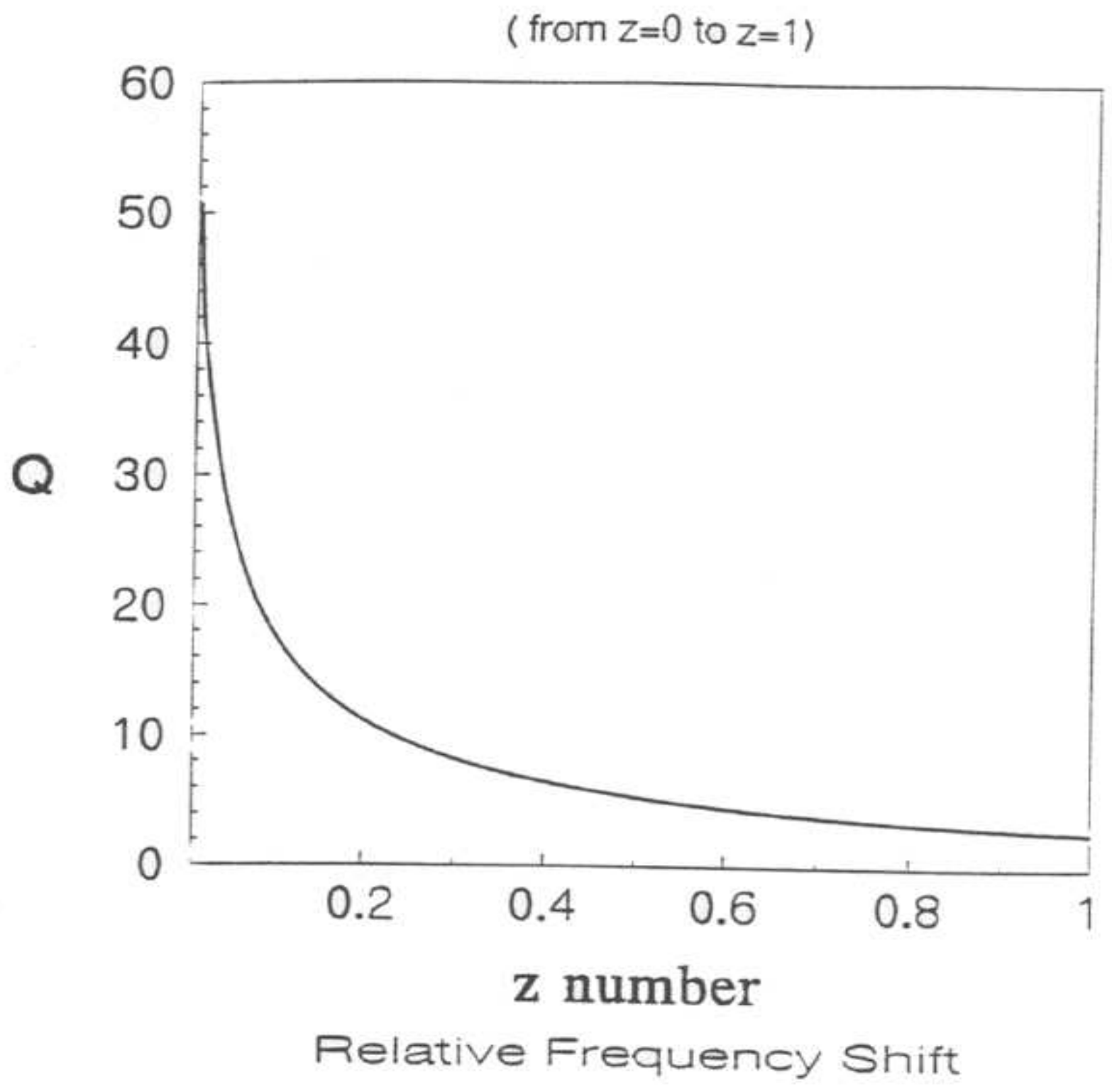

Fig. 2 : Variation of Sharpness of a Spectral line with Relative Frequency Shift ( for low z) 


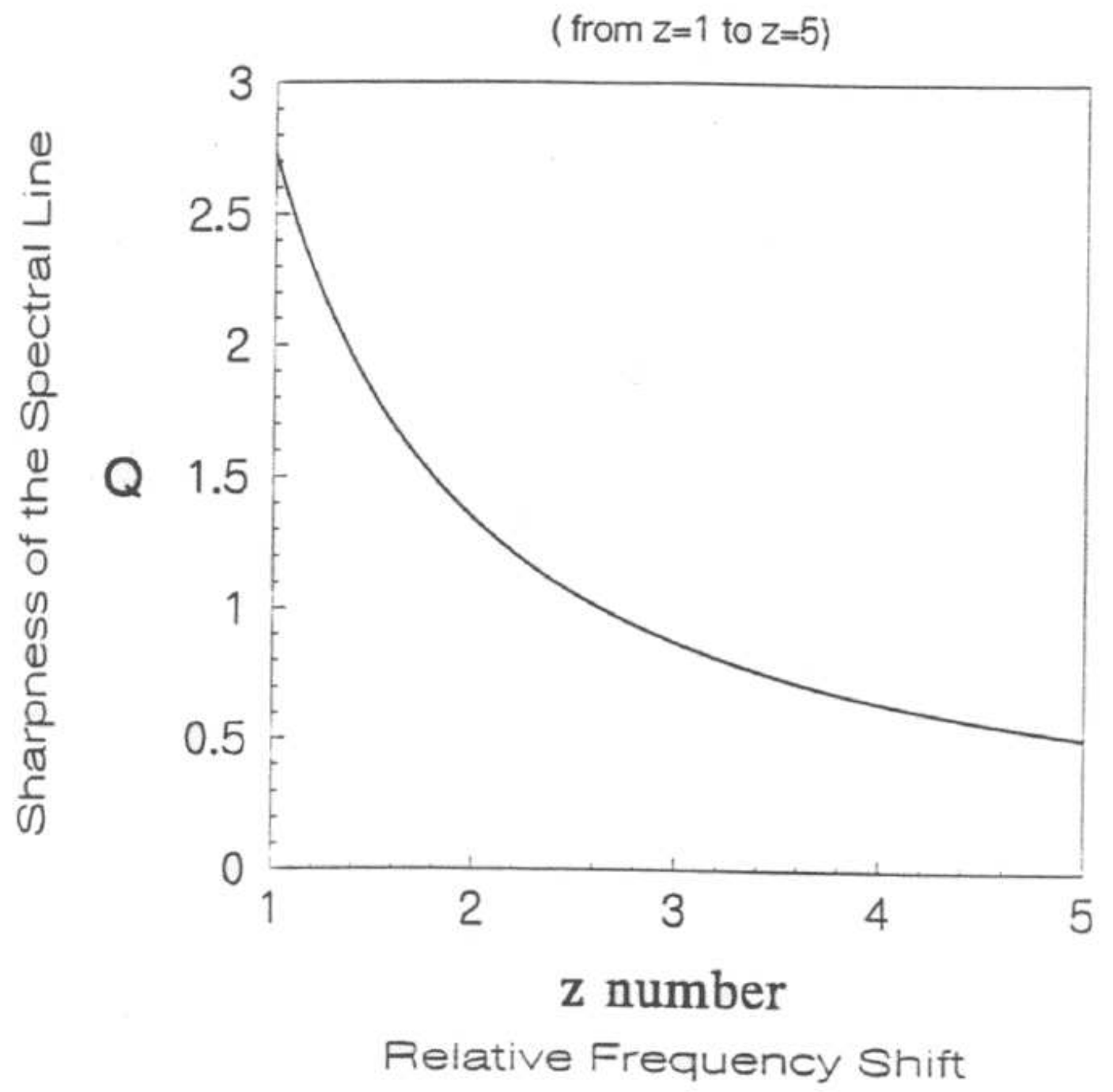

Fig. 3 : Variation of Sharpness for high $\mathrm{z}$ 


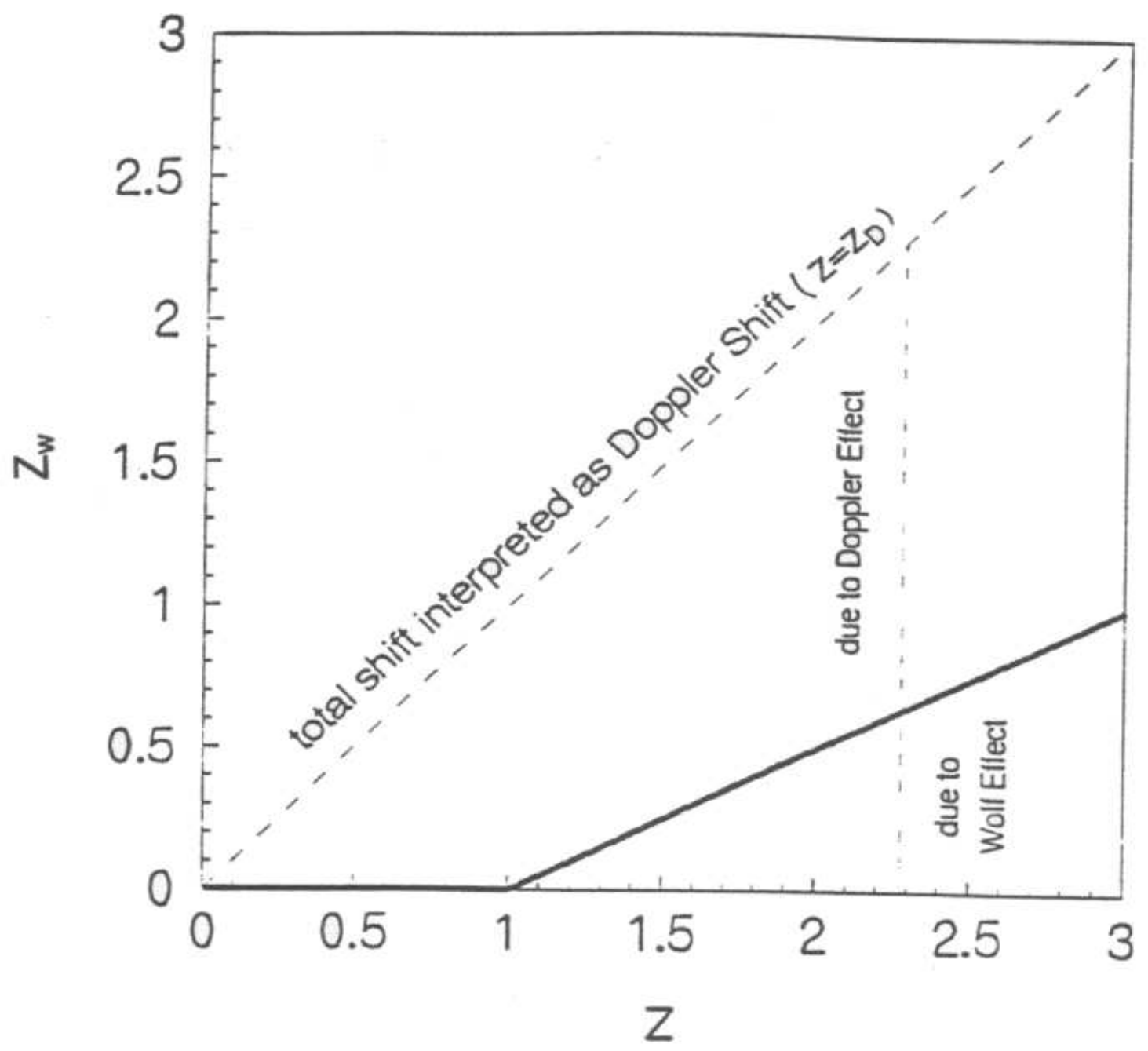

Fig. 4 : Wolf Contribution in Relative Frequency Shift 


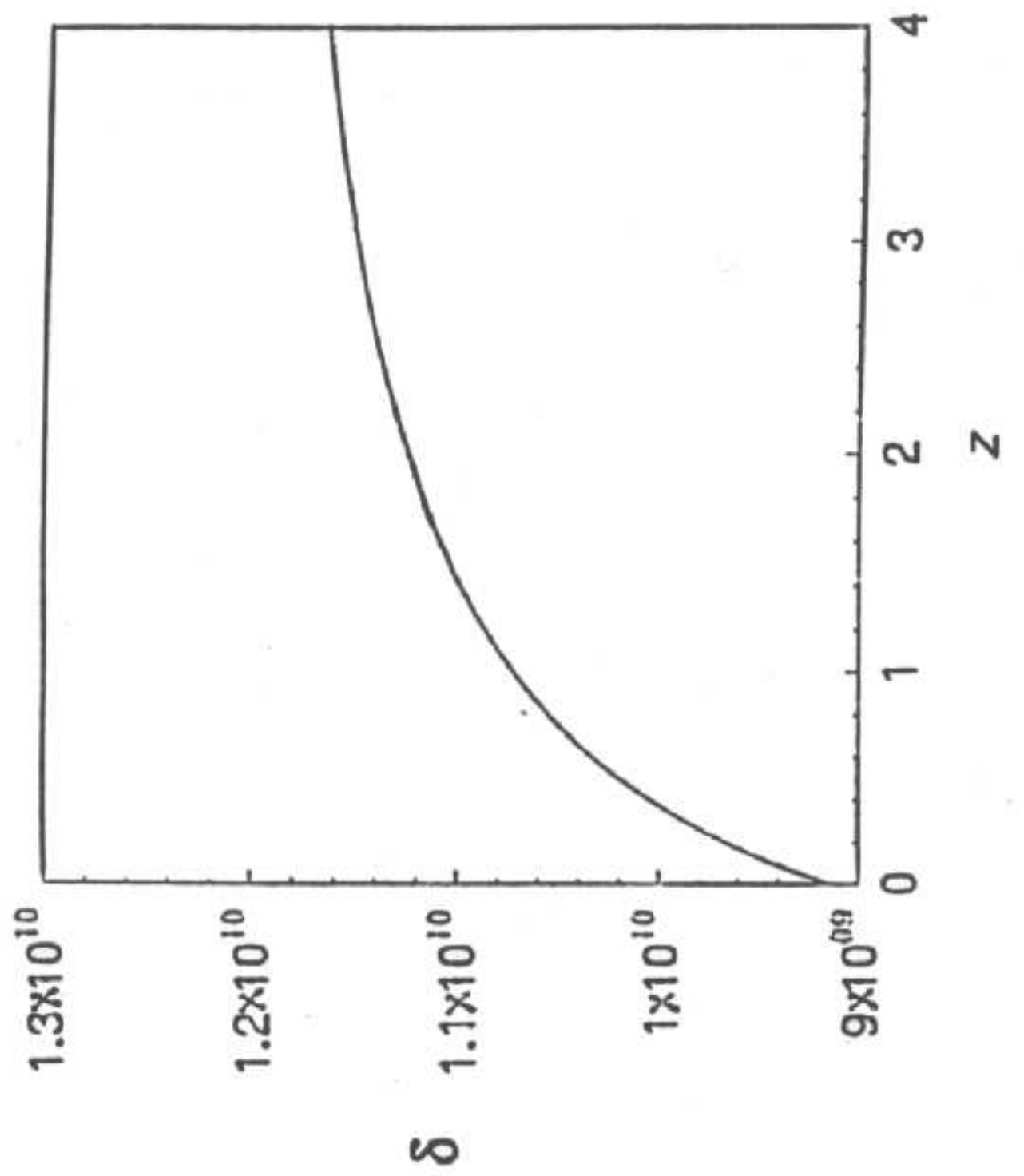

昜

몀

3

4

.일

氜

$>$

n

bi

泣 